\title{
Audiogram in Response to Stimulation Delivered to Fluid Applied to the External Meatus
}

\author{
Miriam Geal-Dor ${ }^{1,2}$, Shai Chordekar ${ }^{1,3}$, Cahtia Adelman ${ }^{1,2}$, \\ Michal Kaufmann-Yehezkely ${ }^{4}$, and Haim Sohmer ${ }^{5}$ \\ ${ }^{1}$ Speech \& Hearing Center, Hadassah University Medical Center, Jerusalem, Israel \\ ${ }^{2}$ Department of Communication Disorders, Hadassah Academic College, Jerusalem, Israel \\ ${ }^{3}$ Department of Communication Disorders, Sackler Faculty of Medicine, Tel Aviv University, Tel Aviv, Israel \\ ${ }^{4}$ Department of Otorhinolaryngology/Head \& Neck Surgery, Hadassah University Medical Center, Jerusalem, Israel \\ ${ }^{5}$ Department of Medical Neurobiology, Hebrew University-Hadassah Medical School, Jerusalem, Israel
}

$\begin{array}{ll}\text { Received } & \text { October 7, 2019 } \\ \text { Revised } & \text { December 11, 2019 } \\ \text { Accepted } & \text { December 23, } 2019\end{array}$

Address for correspondence Haim Sohmer, PhD

Department of Medical Neurobiology, Hebrew University-Hadassah

Medical School, POB 12272,

Jerusalem 91120, Israel

Tel $+972-2-6758385$

Fax +972-2-6439736

E-mail haims@ekmd.huji.ac.il
Background and Objectives: Hearing can be elicited in response to vibratory stimuli delivered to fluid in the external auditory meatus. To obtain a complete audiogram in subjects with normal hearing in response to pure tone vibratory stimuli delivered to fluid applied to the external meatus. Subjects and Methods: Pure tone vibratory stimuli in the audiometric range from 0.25 to $6.0 \mathrm{kHz}$ were delivered to fluid applied to the external meatus of eight participants with normal hearing ( $15 \mathrm{~dB}$ or better) using a rod attached to a standard clinical bone vibrator. The fluid thresholds obtained were compared to the air conduction (AC), bone conduction (BC; mastoid), and soft tissue conduction (STC; neck) thresholds in the same subjects. Results: Fluid stimulation thresholds were obtained at every frequency in each subject. The fluid and STC (neck) audiograms sloped down at higher frequencies, while the AC and BC audiograms were flat. It is likely that the fluid stimulation audiograms did not involve AC mechanisms or even, possibly, osseous BC mechanisms. Conclusions: The thresholds elicited in response to the fluid in the meatus likely reflect a form of STC and may result from excitation of the inner ear by the vibrations induced in the fluid. The sloping fluid audiograms may reflect transmission pathways that are less effective at higher frequencies.

J Audiol Otol 2020;24(2):79-84

KEY WORDS: Audiogram · Osseous · Meatus · Bone conduction · Fluid

\section{Introduction}

Hearing is usually elicited by air conduction (AC) in which sound is conducted to the external ear by air pressures. Bone conduction (BC) elicits hearing when a bone vibrator is applied to the skin overlying the skull bone, and induces vibrations of the underlying bone, which are then conducted to the bony external, middle, and inner ears. Soft tissue conduction (STC) is an additional mode of audition which is elicited by inducing vibrations in the body through, for example, placing a bone vibrator on multiple sites on the body that are not

This is an Open Access article distributed under the terms of the Creative Commons Attribution Non-Commercial License (https://creativecommons.org/licenses/by-nc/4.0/) which permits unrestricted non-commercial use, distribution, and reproduction in any medium, provided the original work is properly cited. over skull bone. The vibrations induced in the body in this way are conducted through the soft tissues of the body to the ear [1-4], and therefore called STC. These soft tissue vibrations converge on the final stage (end point) in the inner ear, activating it [5].

Several previous studies designed to gain insight into the mechanisms of STC have been conducted on cadavers [6] and on patients with $\mathrm{BC}$ implants [7]. In a study investigating STC mechanisms, auditory thresholds were determined in response to the delivery of auditory frequency vibratory stimuli to fluid applied to the mastoidectomy cavity in post-radical mastoidectomy patients, and to fluid in the external auditory meatus (EAM) in humans with normal hearing [8]. However, that study was limited to the thresholds of 1.0 and $2.0 \mathrm{kHz}$ [8]. A complementary study was conducted in an animal 
model of mastoidectomy [9].

In the present study, pure tone vibratory stimuli at all the audiometric frequencies were delivered to fluid, which had been applied to the external meatus of patients with normal hearing. The thresholds elicited were then compared to those obtained in response to $\mathrm{AC}, \mathrm{BC}$ (at the mastoid), and an STC site at the neck (over the sternocleidomastoid muscle) in the same subjects. This was designed to provide insight into the possible mechanisms and pathways involved in STC, which originate at the sites of stimulation and activate the inner ear and auditory nerve fibers leading to auditory sensation. Therefore, the present study provides a complete "fluid stimulation audiogram" in response to pure tones across the entire audiometric frequency range. Since this type of fluid stimulation can be considered a form of STC, it is a convenient platform to study the properties of STC in normal hearing subjects. The study also serves to complement and expand on the earlier report [8], which was limited to frequencies of 1.0 and $2.0 \mathrm{kHz}$.

\section{Subjects and Methods}

Eight female subjects recruited from a local college (mean \pm standard deviation: age $23.8 \pm 2.17$; range 20 to 27 years) with normal hearing (defined as AC thresholds at or above $15 \mathrm{~dB}$ $\mathrm{HL}$ ) at the frequencies $0.25,0.5,1.0,2.0,4.0$, and $6.0 \mathrm{kHz}$ participated in the study. All auditory testing was conducted using the same clinical audiometer (Interacoustics model AC40, Assens, Denmark) in the same audiometric booth by the same audiologist. Prior to the study, an otolaryngologist examined the participants' ears to confirm that they were normal, and that the tympanic membrane was intact. The study was reviewed and approved by the Hebrew University-Hadassah Medical School Ethics Committee and written informed consent was given by all participants (HMO 15-0601).

The participants initially underwent a conventional audiometric evaluation (AC and $\mathrm{BC}$ thresholds) conducted with warble tones (standard at our facility) by an audiologist in the conventional manner (American National Standard Institute S3.31 1978, 1986), using the modified Hughson-Westlake technique. During the experimental part of the study, which followed the initial evaluation, the AC threshold was determined using insert phones (Etymotic Research ER·3A, Elk Grove Village, IL, USA), and the BC threshold was determined using a clinical bone vibrator (Radioear B71, Radioear, New Eagle, PA, USA) with the metal head band (Radioear P3333, Radioear) applied to the mastoid with the standard force of approximately 500 gram $(5 \mathrm{~N})$. The threshold was additionally determined by applying the same bone vibrator to the skin over a soft tissue site at the neck (over the sternocleidomastoid muscle). This neck STC site was chosen due to its accessibility and its use by a previous study [10]. The same bone vibrator was applied to this neck STC site using the same metal $5 \mathrm{~N}$ force head band for each frequency, so that the application force would be similar.

The thresholds to stimulation at the mastoid and at the neck STC sites were obtained while the EAM was occluded with ear plugs (Classic SuperFit 30 AeroCo, E-A-R, Indianapolis, IN, USA) deeply inserted into each ear canal to reduce the possibility that the participants would respond to the air-conducted sound coming from the bone vibrator.

For the fluid stimulation part of the experiment, the head of each subject rested on a pillow with the experimental ear facing upward. Sterile saline was applied to the ear canal and filled to the concha of the external ear. Since the experimental ear was facing upward, the fluid was meant to replace and flush out air from the meatus. A small, thin plastic rod (diameter $3 \mathrm{~mm}$; length $2.5 \mathrm{~cm}$ ) was attached to the bone vibrator so that its vibrations would be conveyed by the rod to the fluid in the canal and the concha, as in previous human experiments [8]. The bone vibrator was held by an independent experimenter, and the rod was dipped into the fluid in the EAM and concha confirmed by visual inspection to be making contact with the fluid alone, without direct contact with the skin of the canal or the concha, i.e., without any application force ( $0 \mathrm{~N}$ force). In addition, the subject was instructed to report if they felt any contact between the rod and the skin. The tip of the rod was placed several millimeters into the fluid. The same standard clinical bone vibrator (Radioear B71) was used to deliver the BC (mastoid), STC (neck), and fluid stimulations.

Using this experimental protocol, four threshold audiograms were conducted in response to stimulation with warble tones at $0.25,0.5,1.0,2.0,4.0$, and $6.0 \mathrm{kHz}$ delivered to the sites AC, BC (mastoid), STC neck site, and fluid in the EAM, and compared. In the contralateral ear an insert earphone presented appropriate $40 \mathrm{~dB}$ SL narrow band noise in all conditions (i.e., during AC, BC, STC neck, and fluid stimulation). Two-way analysis of variance (ANOVA) repeated measures were conducted in order to analyze the main effects of the stimulation site (AC, BC, STC, fluid) and frequency $(0.25$, $0.5,1.0,2.0,4.0$, and $6.0 \mathrm{kHz}$ ).

\section{Results}

Thresholds were obtained for all the participants at each of the frequencies delivered to the fluid in the meatus. The mean thresholds ( \pm standard deviation) were elicited in the eight 
participants with normal hearing at each of the four stimulation sites (AC, BC mastoid, neck STC, and fluid in the EAM) and with each of the stimulus frequencies, which are displayed in Fig. 1. The two-way ANOVA revealed a significant effect in stimulation site $[\mathrm{F}(3,191)=43.28, p<0.001]$, and in frequency $[\mathrm{F}(5,191)=17.92, p<0.001]$, with a significant interaction between these two main effects [F $(15,191)=14.94, p<0.001]$. Contrast analysis (Holm-Sidak) demonstrated that the thresholds of the neck STC site and fluid audiograms at the higher frequencies differed significantly from those at the lower frequencies $(\mathrm{t}=8.85 ; \mathrm{t}=5.97 ; \mathrm{t}=10.4 ; \mathrm{t}=7.08, p<0.001)$. As can be seen in Fig. 1, the $\mathrm{AC}$ and $\mathrm{BC}$ audiograms were relatively flat overall. However, the fluid stimulation and neck STC site audiograms slope down to poorer (higher) thresholds at the higher frequencies.

\section{Discussion}

The present study expands on an earlier report in which audio frequency vibratory stimuli were delivered to fluid applied to the mastoidectomy cavity of post-radical mastoidectomy patients and to the EAM in normal hearing subjects [8]. That study was limited to fluid thresholds of 1.0 and $2.0 \mathrm{kHz}$. In this study, we present fluid thresholds across the entire audiometric frequency range of vibratory stimulation at 0.25 , $0.5,1.0,2.0,4.0$, and $6.0 \mathrm{kHz}$ delivered to fluid applied to the EAM of subjects with normal hearing. Thresholds were obtained in each subject and frequency, providing pure tone audiograms for each stimulation mode (AC, BC, STC neck, and fluid). However, the thresholds of the $\mathrm{BC}$ audiograms cannot be statistically compared to the STC neck and fluid

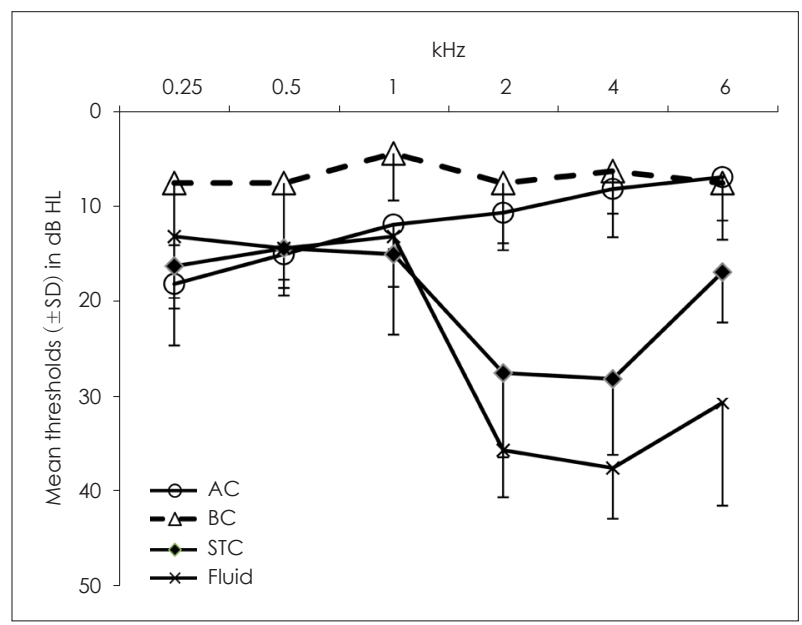

Fig. 1. Mean thresholds $( \pm S D)$ as a function of stimulus frequency to AC, BC (over mastoid), neck STC, and fluid (applied to the EAM) stimulation. AC: air conduction, BC: bone conduction, STC: soft tissue conduction, SD: standard deviation, EAM: external auditory meatus. threshold audiograms. This is because even though the same bone vibrator was used to elicit the BC, STC neck, and fluid thresholds, the bone vibrator and audiometer had been calibrated to deliver vibratory stimulation to the bony mastoid, which presents a higher mechanical impedance, while the other sites (STC neck and fluid) also elicited by the same bone vibrator, presented lower impedance to the bone vibrator. Therefore, the only statistical comparisons that can be used are those that show that the $\mathrm{AC}$ and $\mathrm{BC}$ audiograms were flat, and the STC neck and fluid audiograms sloped down at higher frequencies.

The established view is that $\mathrm{AC}$ and $\mathrm{BC}$ stimulation elicit hearing by the initiation of a pressure difference across the cochlear partition, producing passive displacements of the basilar membrane (a traveling wave), which then activate the outer hair cells (the cochlear amplifier), contributing to active displacements [11]. It has been shown that the administration of drugs which depress the cochlear amplifier (salicylic acid and furosemide) lead to similar AC, BC, and STC threshold elevations [12]. Furthermore AC, BC, and STC hearing mechanisms all interact with each other, which is possible only if they share a common pattern of mechanical activity [13]. Therefore, the final stage of hearing elicited by each mode of stimulation delivered at threshold intensities in the present study (AC, BC, STC neck, and fluid) could all involve similar basilar membrane displacements.

How could the vibrations induced in the fluid applied to the EAM at threshold intensities in normal hearing subjects initiate such basilar membrane displacements?

\section{Possible AC mechanisms}

One consideration is an AC related mechanism, in which either air conducted sounds coming from the bone vibrator, or the vibratory stimuli induced in the fluid in the meatus by the bone vibrator, give rise to vibrations of the tympanic membrane. However, the constant, static pressure of the fluid applied to the meatus and resting on the tympanic membrane, blocking the meatus, would attenuate AC sound. In addition, the fluid on the tympanic membrane would dampen any dynamic vibrations of the tympanic membrane that were potentially induced by the sound. Therefore, the responses obtained in the present study at threshold intensities are likely not due to the initiation of AC (tympanic membrane) related mechanisms.

\section{Possible osseous BC mechanisms}

Another possible consideration is that the fluid thresholds obtained in the present study could be initiated through osseous BC mechanisms. In other words, the magnitude of the vi- 
brations, which were induced in the fluid applied to the EAM (which were threshold in intensity), could have led to vibrations of skull bone, which were then conducted along the bone to the temporal bone. The generally accepted view is that hearing by means of osseous $\mathrm{BC}$ mechanisms is elicited when the clinical bone vibrator is pressed with a static force of approximately 500 gram to the skin at the mastoid or forehead directly overlying skull bone. The vibrations induced in the underlying bone are conducted along skull bone to the temporal bone, leading to vibrations of the bony walls of the external, middle, and inner ears [14-16]. As a result, the following four parallel mechanisms of osseous BC are induced: 1) the occlusion effect, where vibrations of the wall of the EAM give rise to air pressures in the occluded meatus which drive the tympanic membrane, as in AC; 2) middle ear ossicular chain inertia: an inertia-related phase lag of the vibrations of the ossicles with respect to the vibrations of the middle ear wall; 3 ) inertia of inner ear fluid; or 4) distortion (compression-expansion) of the bony wall of the inner ear [14-16]. Together, these four osseous BC mechanisms lead to pressure differences across the basilar membrane and to a passive traveling wave, similar to AC hearing [14-16]. Therefore, threshold intensity vibratory stimulation delivered to the fluid without any application force could have initiated vibrations of skull bone, leading to these four osseous BC mechanisms. Furthermore, the fluid in the meatus was not in direct contact with bone, but rather with the soft tissues lining the external meatus.

In a previous study conducted on post radical mastoidectomy patients (in whom the tympanic membrane and the ossicular chain had been surgically removed) in addition to normal subjects, the fluid stimulation thresholds in the patient group were similar to those in the normal hearing subject group at 1.0 and $2.0 \mathrm{kHz}$ [8]. However, in the post radical mastoidectomy patients, due to the surgical removal of the tympanic membrane and the ossicular chain, the occlusion effect and the ossicle inertia mechanisms would not be possible. Furthermore, the removal of the ossicular chain together with the tympanic membrane would greatly reduce the impedance acting on the oval window, making its compliance, and hence its displacement, more similar to that of the round window. In such a situation, the two inner ear osseous BC mechanisms (fluid inertia and distortion: compression-expansion of the wall of the inner ear) should also be less effective. Therefore, it was expected that each of the four osseous BC mechanisms would have reduced efficacy in post radical mastoidectomy patients. Nevertheless, in several post radical mastoidectomy and subtotal petrosectomy patients in whom the tympanic membrane and the ossicles had been removed, normal BC thresholds were obtained [17], and in some of them, the STC neck thresholds were similar to those in normal subjects (personal communication: MG-D, MK-Y). Therefore, the finding that the fluid stimulation thresholds at 1.0 and 2.0 $\mathrm{kHz}$ in the post mastoidectomy patients were similar to those in the normal subjects, coupled with the normal BC and STC (neck) thresholds in many radical mastoidectomy patients, could mean that the fluid stimulation thresholds at 1.0 and $2.0 \mathrm{kHz}$ in the post mastoidectomy patients and in the normal subjects in the previous study [8] may not have involved osseous BC mechanisms. This also supports the notion that the fluid stimulation threshold audiograms in the present study conducted on subjects with normal hearing may not have been elicited by osseous BC mechanisms.

\section{Acoustic impedance}

In addition, it should be difficult for threshold intensity pressures induced in the fluid applied to the external meatus to give rise to vibrations of skull bone, leading to the osseous BC mechanisms. This intuitive concept can be expressed in physical principles: the acoustic impedance (defined as the density of a medium multiplied by the velocity of sound in the medium) of the soft tissues of the body and tissue fluids are similar to each other ( soft tissue $=1.62 \times 10^{6} \mathrm{~kg} / \mathrm{m}^{2} \mathrm{sec}$; fluid $=1.52 \times$ $\left.10^{6} \mathrm{~kg} / \mathrm{m}^{2} \mathrm{sec}\right)$ and are very different from that of bone $(7.8 \times$ $\left.10^{6} \mathrm{~kg} / \mathrm{m}^{2} \mathrm{sec}\right)$. Therefore, the threshold intensity vibrations induced in the fluid, skin, and other soft tissues, can be transmitted through a series of soft tissues and fluids in the body to the inner ear fluids $[5,18,19]$. However, they would be partially reflected at the boundary between the soft tissues and the more compact, dense, and solid skull bone. At supra-threshold intensities (approximately $10 \mathrm{~dB}$ higher), however, osseous BC mechanisms may be involved. It is therefore possible that the hair cells of the inner ear may be activated at the threshold directly by the vibrations induced in the EAM fluid, and not by the induction of osseous BC mechanisms. In fact, other forms of fluid stimulation in experimental animals also elicited threshold auditory brainstem evoked responses, but with the apparent absence of osseous BC mechanisms. These experiments were conducted under conditions in which a traveling wave along the basilar membrane may not have been possible (for example, following the immobilization of the ossicular chain, discontinuity of the ossicular chain, and fixation of the cochlear windows, so that bulk fluid flow between the two immobilized windows) would not be possible. Nevertheless, $\mathrm{BC}$ thresholds did not change, while in these same animals, threshold auditory responses could be elicited in response to the delivery of vibratory stimulation to a pool of saline in the surgical region [20], to the brain and cerebrospinal 
fluid in the cranial cavity, and to the fluid (saline) applied to the middle ear cavity [21]. The severe impediments to the osseous BC mechanisms of ossicular chain inertia, and to the inner ear mechanisms of fluid inertia and distortion (compression-expansion) of the cochlear wall resulting from the changes induced in the load impedance of the oval window, would further hinder the initiation of a traveling wave. However, auditory responses could be elicited in response to the delivery of vibrations to fluid, as in the present study. The present experimental results, together with the consideration of BC mechanisms and the acoustic impedances of body tissues, have provided evidence that hearing at threshold may not involve those osseous BC mechanisms (which are based on the induction of actual bone vibrations) in these forms of STC. Nevertheless, at somewhat higher intensities, osseous BC mechanisms may be induced [7].

The results of the present fluid stimulation audiogram study show that both the AC and BC audiograms elicited by bone vibrator stimulation at the mastoid are relatively flat. On the other hand, the thresholds of the STC neck site and the fluid stimulation in the EAM audiograms slope down at the higher frequencies. Thus, the findings that the $\mathrm{BC}$ and $\mathrm{AC}$ audiograms were flat while the STC neck and fluid audiograms (the latter of which is also a form of STC) slope down at higher frequencies, may be evidence that the STC pathways in general may be less efficient at higher frequencies, which may reflect some inherent property of the STC pathways.

While others have conducted similar fluid stimulation studies, they have done so for completely different reasons. Tabuchi, et al. [22] conducted a "liquid test" in order to differentiate between ossicular fixation and ossicular discontinuity. Nishimura, et al. [23] applied water to the ear canal in order to analyze what they refer to as "cartilage conduction." In both studies, pure tone stimuli were delivered to fluid in the canal, and lower (better) thresholds were found at the lower frequencies, similar to the results of the present study.

Future studies are necessary to elucidate the final stage of hearing resulting from STC stimulation and to assess the mechanism responsible for the sloping audiograms seen during fluid and the STC stimulation. In addition, fluid stimulation audiograms should be compared to the more standard AC and $\mathrm{BC}$ audiograms in large groups of patients with different types of hearing loss in order to assess whether fluid stimulation audiograms reflect lesions of the inner ear (sensori-neural hearing loss) or of the middle ear (conductive hearing loss).

In conclusion, a complete threshold audiogram can be elicited in response to the delivery of pure tone vibratory stimulation to fluid applied to the EAM. Analysis of the results of this fluid stimulation study contributes to a better understand-

ing of the mechanisms of STC at threshold intensities.

\section{Acknowledgments}

The participation of Miriam Geal-Dor was supported by the Newman Fund for audiological research.

We would like to thank the Newman Fund for audiological research (The fund had no involvement in the study design, in the collection, analysis, and interpretation of data; in the writing of the manuscript; and in the decision to submit the manuscript for publication).

\section{Conflicts of interest}

The authors have no financial conflicts of interest.

\section{Author Contributions}

Conceptualization: Haim Sohmer. Data curation: Miriam Geal-Dor. Formal analysis: Shai Chordekar. Methodology: Miriam Geal-Dor. Project administration: Haim Sohmer. Supervision: Haim Sohmer. Validation: Shai Chordekar. Writing — original draft: Haim Sohmer and Miriam Geal-Dor. Writing - review \& editing: Miriam Geal-Dor, Shai Chordekar, Cahtia Adelman, Michal Kaufmann-Yehezkely, and Haim Sohmer.

\section{ORCID iDs}

\section{Miriam Geal-Dor}

Shai Chordekar

Cahtia Adelman

Michal Kaufmann-Yehezkely

Haim Sohmer

\section{REFERENCES}

1) Ravicz ME, Melcher JR. Isolating the auditory system from acoustic noise during functional magnetic resonance imaging: examination of noise conduction through the ear canal, head, and body. J Acoust Soc Am 2001;109:216-31.

2) Berger EH, Kieper RW, Gauger D. Hearing protection: surpassing the limits to attenuation imposed by the bone-conduction pathways. J Acoust Soc Am 2003;114:1955-67.

3) Kaufmann M, Adelman C, Sohmer H. Mapping at sites on bone and soft tissue on the head, neck and thorax at which a bone vibrator elicits auditory sensation. Audiol Neurotol Extra 2012;2:9-15.

4) Adelman C, Yehezkely MK, Chordekar S, Sohmer H. Relation between body structure and hearing during soft tissue auditory stimulation. Biomed Res Int 2015;2015:172026.

5) Sohmer H. Soft tissue conduction: review, mechanisms, and implications. Trends Hear 2017;21:2331216517734087.

6) Sim JH, Dobrev I, Gerig R, Pfiffner F, Stenfelt S, Huber AM, et al. Interaction between osseous and non-osseous vibratory stimulation of the human cadaveric head. Hear Res 2016;340:153-60.

7) Chordekar S, Perez R, Adelman C, Sohmer H, Kishon-Rabin L. Does hearing in response to soft-tissue stimulation involve skull vibrations? A within-subject comparison between skull vibration magnitudes and hearing thresholds. Hear Res 2018;364:59-67.

8) Ronen O, Geal-Dor M, Kaufmann-Yehezkely M, Perez R, Chordekar S, Adelman C, et al. Inner ear excitation in normal and postmastoidectomy participants by fluid stimulation in the absence of air- and bone-conduction mechanisms. J Am Acad Audiol 2017;28:152-60.

9) Perez R, Adelman C, Sohmer H. Fluid stimulation elicits hearing in the absence of air and bone conduction--an animal study. Acta Otolaryngol 2016;136:351-3.

10) Adelman C, Sohmer H. Thresholds to soft tissue conduction stimulation compared to bone conduction stimulation. Audiol Neurootol 
2013;18:31-5.

11) Oghalai JS. The cochlear amplifier: augmentation of the traveling wave within the inner ear. Curr Opin Otolaryngol Head Neck Surg 2004;12:431-8.

12) de Jong M, Perez R, Adelman C, Chordekar S, Rubin M, Kriksunov $\mathrm{L}$, et al. Experimental confirmation that vibrations at soft tissue conduction sites induce hearing by way of a new mode of auditory stimulation. J Basic Clin Physiol Pharmacol 2011;22:55-8.

13) Adelman C, Fraenkel R, Kriksunov L, Sohmer H. Interactions in the cochlea between air conduction and osseous and non-osseous bone conduction stimulation. Eur Arch Otorhinolaryngol 2012;269:425-9.

14) Stenfelt S, Goode RL. Bone-conducted sound: physiological and clinical aspects. Otol Neurotol 2005;26:1245-61.

15) Stenfelt S. Acoustic and physiologic aspects of bone conduction hearing. Adv Otorhinolaryngol 2011;71:10-21.

16) Tonndorf J. Bone conduction. Studies in experimental animals. Acta Otolaryngol 1966;Suppl 213:1-132.

17) Yehezkely MK, Grinblat G, Dor MG, Chordekar S, Perez R, Adelman $\mathrm{C}$, et al. Implications for bone conduction mechanisms from thresholds of post radical mstoidectomy and subtotal petrosectomy patients. J Int Adv Otol 2019;15:8-11.
18) Wever EG, Lawrence M. The function of the middle ear. In: Wever EG, Lawrence M, editors. Physiological Acoustics Princeton. Princeton, NJ: Princeton University Press;1954. p.69-78.

19) Baun J. Interaction with soft tissue. In: Baun J, editor. Physical Principles of General and Vascular Sonography. San Francisco, CA: ProSono Publishing;2004. p.28-41.

20) Perez R, Adelman C, Sohmer H. Bone conduction activation through soft tissues following complete immobilization of the ossicular chain, stapes footplate and round window. Hear Res 2011;280:82-5.

21) Perez R, Adelman $C$, Chordekar S, Ishai R, Sohmer H. Air, bone and soft tissue excitation of the cochlea in the presence of severe impediments to ossicle and window mobility. Eur Arch Otorhinolaryngol 2015;272:853-60.

22) Tabuchi K, Murashita H, Okubo H, Takahashi K, Wada T, Hara A. Preoperative evaluation of ossicular chain abnormality in patients with conductive deafness without perforation of the tympanic membrane. Arch Otolaryngol Head Neck Surg 2005;131:686-9.

23) Nishimura T, Hosoi H, Saito O, Miyamae R, Shimokura R, Yamana$\mathrm{ka} \mathrm{T}$, et al. Cartilage conduction is characterized by vibrations of the cartilaginous portion of the ear canal. PLoS One 2015;10:e120135. 